\title{
On graph-theoretical invariants of combinatorial manifolds
}

\author{
Alberto Cavicchioli Fulvia Spaggiari \\ Dipartimento di Scienze Fisiche, Informatiche e Matematiche \\ Universitá di Modena e Reggio E., via Campi 213/B \\ 41100 Modena, Italy \\ \{alberto.cavicchioli, fulvia.spaggiari\}@unimore.it
}

Submitted: Nov 23, 2017; Accepted: May 23, 2019; Published: Jul 5, 2019

(c) The authors.

\begin{abstract}
The goal of this paper is to give some theorems which relate to the problem of classifying combinatorial (resp. smooth) closed manifolds up to piecewise-linear (PL) homeomorphism. For this, we use the combinatorial approach to the topology of PL manifolds by means of a special kind of edge-colored graphs, called crystallizations. Within this representation theory, Bracho and Montejano introduced in 1987 a nonnegative numerical invariant, called the reduced complexity, for any closed $n$-dimensional PL manifold. Here we consider this invariant, and extend in this context the concept of average order first introduced by Luo and Stong in 1993, and successively investigated by Tamura in 1996 and 1998. Then we obtain some classification results for closed connected smooth low-dimensional manifolds according to reduced complexity and average order. Finally, we answer to a question posed by Trout in 2013 .
\end{abstract}

Mathematics Subject Classifications: 57N15, 57Q15, 05C10

\section{Colored Graphs and Crystallizations}

All spaces and maps will be considered in the PL category, for which we refer to [16]. The definitions and main results of Graph Theory can be found in [10]. For the representation of PL manifolds by means of edge-colored graphs and crystallizations see the survey papers $[1,2,7,9,21]$.

Here we recall the necessary definitions to explain the statements of our main theorem. An $(n+1)$-colored graph $(G, c)$ is a multigraph $G=(V(G), E(G))$, regular of degree $n+1$ (possibly with multiple edges, but without loops), together with a proper edge-coloring $c: E(G) \rightarrow \Delta_{n}=\{i \in \mathbb{Z}: 0 \leqslant i \leqslant n\}$. This means that any two adjacent edges in $G$ are 
differently colored. As usual, $V(G)$ and $E(G)$ denote the vertex set and the edge set of $G$, respectively; $\Delta_{n}$ will be called the color set, and its elements the colors.

The cellular complex $K=K(G)$ associated to $G$ is constructed as follows: (1) for each vertex $v$ of $G$, consider a standard $n$-simplex $\sigma^{n}(v)$, and label its $n+1$ vertices by the colors of $\Delta_{n}$; (2) if $v$ and $w$ are joined in $G$ by an $i$-colored edge, then identify the $(n-1)$-faces of $\sigma^{n}(v)$ and $\sigma^{n}(w)$ opposite the vertex labelled by $i \in \Delta_{n}$, so that equally labelled vertices coincide. The complex $K(G)$ is not a classical simplicial complex for two simplexes may meet in more than a single face. On the other hand, it is a pseudocomplex in the sense of [11], p.49. This means that any simplex of $K(G)$ is canonically isomorphic to a standard one, and the intersection of two simplexes can be either empty or a union of common faces. By construction, the graph $G$ can be thought as the 1-skeleton of the dual cellular complex of $K(G)$.

Let $M^{n}$ be a closed connected PL (or, smooth) $n$-manifold. We say that $(G, c)$ represents $M$ if $M$ is PL homeomorphic to the space underlying $K(G)$. A crystallization of $M$ is an $(n+1)$-colored graph $(G, c)$ representing $M$ such that $K(G)$ has exactly $n+1$ vertices (which we shall always assume to be colored by the elements of $\Delta_{n}$ ). In this case, $K(G)$ is called a contracted triangulation of $M$.

The following is a famous theorem of Pezzana (for the proof see, for example, [7]):

Theorem 1. Every closed connected PL (or, smooth) n-manifold admits a crystallization, that is, it has a contracted triangulation.

Let $M$ be a closed connected PL $n$-manifold, $(G, c)$ a crystallization of $M$ (with color set $\Delta_{n}$ ), and $K=K(G)$ the associated contracted triangulation of $M$. If $\Gamma \subset \Delta_{n}$, then $g_{\Gamma}$ represents the number of connected components of the partial subgraph $G_{\Gamma}=$ $\left(V(G), c^{-1}(\Gamma)\right)$. If $\Gamma=\{i, j\}$ (resp. $\{r, s, t\}$ and $\{h, k, r, s\}$ ), then $g_{\Gamma}$ will be simply written as $g_{i j}$ (resp. $g_{r s t}$ and $g_{h k r s}$ ). Let $p$ denote the order of $G$, i.e., the number of vertices in the graph. We always assume that $\left\{v_{i}: i \in \Delta_{n}\right\}$ is the vertex set of $K$, and that $v_{i}$ corresponds to $G_{\hat{i}}$, where $\widehat{i}=\Delta_{n} \backslash\{i\}$.

Theorem 2. An $(n+1)$-colored graph $(G, c)$ is a crystallization of a closed connected $\mathrm{PL} n$-manifold if and only if every partial subgraph $G_{\hat{i}}$ is connected and represents the $(n-1)$-sphere, for every $i \in \Delta_{n}$.

Let $q_{h}(K)$ denote the number of $h$-simplexes in $K$, for any $h \in \Delta_{n}$. For any $\Gamma \subset \Delta_{n}$ with cardinality $h, g_{\Gamma}$ is also the number of $(n-h)$-simplexes of $K=K(G)$ whose vertices are labelled by colors in $\Delta_{n} \backslash \Gamma$.

Using crystallizations, we can associate some numerical invariants to any closed connected PL manifold. See, for example, $[2,3,4,7]$. Here we are interested in two of them, called reduced complexity and average order, which will be presented in the next two sections together with new results about characterizations of certain PL manifolds, up to PL homeomorphisms. 


\section{Reduced complexity}

Let $M$ be a closed connected PL $n$-manifold. Following [2], we define the complexity $\mathbf{c}(M)$ of $M$ as the minimum number of $n$-simplexes which a contracted triangulation of $M$ must have. In other words, $\mathbf{c}(M)$ is the minimum order of a crystallization which represents $M$. Since any crystallization has at least two vertices, it was defined in [2] the reduced complexity of $M$ as $\widetilde{\mathbf{c}}(M)=\mathbf{c}(M)-2$. This combinatorial invariant gives a finite-to-one map from the class of closed connected PL $n$-manifolds to the set of nonnegative even integers. Of course, the only $n$-manifold of reduced complexity zero is the standard $n-$ sphere $\mathbb{S}^{n}$. For any closed connected surface $M$, we have $\widetilde{\mathbf{c}}(M)=4-2 \chi(M)$, where $\chi(M)$ is the Euler characteristic of $M$ (see Theorem 3.13 of [2]). Thus the reduced complexity can be regarded as a generalization of the Euler characteristic. Moreover, it has the nice property of classifying manifolds up to a finite ambiguity. More precisely, if we know a closed connected manifold $M$ has a specific value of reduced complexity, then there are only finitely many topological types possible for $M$.

The classification of all closed connected 3-manifolds with reduced complexity less than or equal to 28 was given in [5] and [12], §5, by using computer algorithms. There are exactly sixty-nine of such manifolds. Among them, there are $\mathbb{S}^{3}, \mathbb{S}^{1} \times \mathbb{S}^{2}$, twenty-eight lens spaces, the six Euclidean orientable 3-manifolds, and sixteen quotients of $\mathbb{S}^{3}$ by the action of their finite (non-cyclic) fundamental groups.

The complete classification of all closed connected PL 4-manifolds up to reduced complexity 14 was obtained in [6]. To clarify the next statement, we first explain the twisted bundle notation. Let $\mathbb{S}^{1} \times \mathbb{S}^{n-1}\left(\right.$ resp. $\left.\mathbb{S}^{1} \times \mathbb{S}^{n-1}\right)$ denote the orientable (resp. non orientable or twisted) $\mathbb{S}^{n-1}$-bundle over $\mathbb{S}^{1}$.

Then the main theorem of [6] is the following:

Theorem 3. (a) There are no closed connected 4-manifolds $M$ of reduced complexity $0<\widetilde{\mathbf{c}}(M)<6$. The unique closed connected 4 -manifold of reduced complexity 6 is the complex projective plane $\mathbb{C} P^{2}$.

(b) $M^{4}$ be a closed connected 4-manifold. If $\widetilde{\mathbf{c}}(M)=8$, then $M$ is PL homeomorphic to either $\mathbb{S}^{1} \times \mathbb{S}^{3}$ or $\mathbb{S}^{1} \times \mathbb{S}^{3}$. There are no closed connected 4 -manifolds of reduced complexity 10 .

(c) The unique closed connected prime 4-manifold of reduced complexity 12 is the topological product $\mathbb{S}^{2} \times \mathbb{S}^{2}$.

(d) The unique closed connected prime 4-manifold of reduced complexity 14 is the real projective 4-space $\mathbb{R} P^{4}$.

In [8], it was given the classification of the closed connected PL (or, smooth) 5manifolds up to reduced complexity 20. This gives combinatorial characterizations of $\mathbb{S}^{1} \times \mathbb{S}^{4}, \mathbb{S}^{1} \times \mathbb{S}^{4}$ and $\mathbb{S}^{2} \times \mathbb{S}^{3}$ among closed PL 5-manifolds.

More precisely, the main result of [8] is the following: 
Theorem 4. (a) The only reduced complexity zero 5-manifold is $\mathbb{S}^{5}$, and there are no closed connected 5-manifolds $M$ of reduced complexity $0<\widetilde{\mathbf{c}}(M)<10$. The only closed connected 5-manifolds of reduced complexity 10 are $\mathbb{S}^{1} \times \mathbb{S}^{4}$ and $\mathbb{S}^{1} \times \mathbb{S}^{4}$.

(b) There are no closed connected 5 -manifolds $M$ of reduced complexity $10<\widetilde{\mathbf{c}}(M)<20$. The only closed connected spin 5-manifolds of reduced complexity 20 are $\mathbb{S}^{2} \times \mathbb{S}^{3}$ and the connected sums $N_{1} \# N_{2}$, where each $N_{i}, i=1,2$, is either $\mathbb{S}^{1} \times \mathbb{S}^{4}$ or $\mathbb{S}^{1} \times \mathbb{S}^{4}$.

Further results and conjectures concerning with the reduced complexity of triangulated manifolds can be found in the quoted papers.

\section{Average order}

Let $K$ be a simplicial triangulation of a closed connected 3-manifold $M$ with $E_{0}(K)$ edges and $F_{0}(K)$ triangles. Note that we distinguish a simplicial triangulation from a pseudocomplex (or, in general, a cell decomposition) into a union of 3-simplexes, that is, such a cell decomposition is a triangulation when the intersection of any two simplexes is actually a single face of each of them. The order of an edge in $K$ is the number of triangles incident to that edge. The average edge order of $K$ was defined in [13] as

$$
\mu_{0}(K)=\frac{3 F_{0}(K)}{E_{0}(K)}
$$

Luo and Stong showed in [13] that for a closed 3-manifold $M, \mu_{0}(K)$ being small implies that the topology of $M$ is fairly simple and restricts the triangulation $K$. The relations between this quantity and the topology of $M$ were investigated in the quoted paper, and the main result of [13] is stated as follows:

Theorem 5. Let $K$ be any simplicial triangulation of a closed connected 3-manifold $M$. Then

(a) $3 \leqslant \mu_{0}(K)<6$, equality holds if and only if $K$ is the simplicial triangulation of the boundary of a 4-simplex.

(b) For any $\epsilon>0$ there are simplicial triangulations $K_{1}$ and $K_{2}$ of $M$ such that

$$
\mu_{0}\left(K_{1}\right)<4.5+\epsilon \quad \text { and } \quad \mu_{0}\left(K_{2}\right)>6-\epsilon .
$$

(c) If $\mu_{0}(K)<4.5$, then $K$ is a simplicial triangulation of $\mathbb{S}^{3}$.

(d) If $\mu_{0}(K)=4.5$, then $K$ is a simplicial triangulation of $\mathbb{S}^{3}, \mathbb{S}^{1} \times \mathbb{S}^{2}$ or $\mathbb{S}^{1} \times \mathbb{S}^{2}$.

Similar results for 3-manifolds with non-empty boundary were established by Tamura $[17,18]$. For a related study see also the paper of Walkup [22]. This concept was extended 
in [4] to higher dimension, and successively investigated there (see [3] for the 3-dimensional case) for the class of colored triangulations of PL $n$-manifolds.

Let now $K$ be a colored triangulation of a closed connected $n$-manifold $M$, that is, $K$ is a pseudocomplex triangulating $M$, whose vertices are labeled by $\Delta_{n}$ so that the coloring is injective on each $n$-simplex of $K$. For such a colored triangulation $K$, it is natural to define the average $(n-2)$-simplex order of $K$ (see [4]) as

$$
\mu(K)=\frac{n q_{n-1}(K)}{q_{n-2}(K)}
$$

where $q_{k}(K)$ is the number of $k$-simplexes of $K$, for $k=0, \ldots, n$. The following is the main theorem of [4] (which extends that of [3] obtained in dimension 3).

Theorem 6. Let $K$ be any colored triangulation of a closed connected PL $n$-manifold $M^{n}, n \geqslant 3$. Then

(a) $2 \leqslant \mu(K)<6$, equality holds if and only if $K$ is the standard (two $n$-simplexes) colored triangulation of $\mathbb{S}^{n}$.

(b) For any $\epsilon>0$ there exists a colored triangulation $K_{\epsilon}$ of $M$ such that

$$
\mu\left(K_{\epsilon}\right)<\frac{2(n+1)}{(n-1)}+\epsilon
$$

If $n=3$, there exists a colored triangulation $\bar{K}_{\epsilon}$ of $M$ such that $\mu\left(\bar{K}_{\epsilon}\right)>6-\epsilon$.

(c) If $\mu(K)<\frac{2(n+1)}{(n-1)}$, then $K$ is a colored triangulation of $\mathbb{S}^{n}$.

(d) For $3 \leqslant n \leqslant 5$, if $\mu(K)=\frac{2(n+1)}{(n-1)}$, then $K$ is a colored triangulation of one of the following $n$-manifolds: $\mathbb{S}^{n}, \mathbb{S}^{1} \times \mathbb{S}^{n-1}, \mathbb{S}^{1} \times \mathbb{S}^{n-1}$ or (for $n=3$ ) the real projective space $\mathbb{R} P^{3}$.

Remark. Note that $\mu_{0}$ in (1) and $\mu$ in (2) have essentially the same definition, but they assume in general quite different values (this is the reason to use distinct notations). In fact, the former is defined by using the class of simplicial triangulations, while the latter arises from the different class of colored pseudocomplexes.

Now we recall the definition of a further combinatorial invariant of closed connected PL $n$-manifolds. Let $K$ be a contracted triangulation of a closed connected PL $n$-manifold $M$, and $\mu(K)$ the average $(n-2)$-simplex order of $K$. By [7] the (contracted) average $(n-2)-$ simplex order of $M$ (in short, the average order), written $\mu^{-}(M)$, is the smallest $\mu(K)$ for a contracted triangulation $K$ of $M$. The motivation for introducing this invariant comes from the 2-dimensional case (see also [13]). Suppose we have a contracted triangulation $K$ of a closed connected surface $M$. Then $q_{0}(K)=3, q_{1}(K)=(3 p) / 2$ and $q_{2}(K)=p$ for $p \geqslant 2$ even. Hence $\mu^{-}(M)=p=6-2 \chi(M)$ by a Euler characteristic calculation. 
Therefore, the average vertex order being less than 6 , equal to 6 , or greater than 6 corresponds to $M$ having a spherical, Euclidean, or hyperbolic structure, respectively.

It is also convenient to introduce a new numerical invariant for a closed connected PL $n$-manifold $M$. Let $\mu^{+}(M)$ denote the maximum $\mu(K)$ for a contracted triangulation $K$ of $M$. Of course, if $M$ is a closed connected surface, then $\mu^{-}(M)=\mu^{+}(M)=6-2 \chi(M)$.

In dimension 3, we are able to improve Theorem II of [3] in order to get the following result:

Theorem 7. Let $M$ be a closed connected PL 3-manifold. Then the reduced complexity and the average order of $M$ are linked by the following formula:

$$
\mu^{-}(M)=\frac{6 \widetilde{\mathbf{c}}(M)+12}{\widetilde{\mathbf{c}}(M)+6} .
$$

Furthermore, we have:

(a) If $2 \leqslant \mu^{-}(M)<4$, then $M$ is PL homeomorphic to a 3-sphere. If $\mu^{-}(M)=4$, then $M$ is $\mathrm{PL}$ homeomorphic to either $\mathbb{S}^{1} \times \mathbb{S}^{2}, \mathbb{S}^{1} \times \mathbb{S}^{2}$ or $\mathbb{R} P^{3}$.

(b) Suppose now $M$ prime and orientable. Then we have:

- If $4<\mu^{-}(M)<5$, then $M$ is PL homeomorphic to one of the lens spaces $L(3,1)$, $L(4,1)$, and $L(5,2)$, or the quaternionic space $\mathbb{S}^{3} /<2,2,2>$.

- If $\mu^{-}(M)=5$, then $M$ is $\mathrm{PL}$ homeomorphic to $L(5,1), L(7,2), L(8,3)$, or the prism manifold $\mathbb{S}^{3} /<3,2,2>$.

- For $5<\mu^{-}(M)<5.2$, there are six lens spaces, seven spherical manifolds with finite noncyclic fundamental groups, and four euclidean fibered manifolds (see the Appendix for more detailed information).

Proof. If $p$ denotes the number of tetrahedra in a contracted triangulation of $K$, then $q_{2}(K)=2 p$. Since $\chi(M)=0$ and $q_{0}(K)=4$, we get $q_{1}(K)=4+q_{2}(K)-p=4+p$. This gives

$$
\mu(K)=\frac{3 q_{2}(K)}{q_{1}(K)}=\frac{6 p}{4+p}
$$

hence $2 \leqslant \mu(K)<6$. The derivative of the function $f(p)=(6 p) /(4+p)$ with respect to the variable $p$ is given by

$$
f^{\prime}(p)=\frac{24}{(4+p)^{2}}>0
$$

Thus the function $f(p)$ is increasing with respect to $p$. Since $\widetilde{\mathbf{c}}(M)+2 \leqslant p$, we get

$$
2 \leqslant \mu^{-}(M)=\frac{6 \widetilde{\mathbf{c}}(M)+12}{6+\widetilde{\mathbf{c}}(M)}<6 .
$$

If $4<\mu^{-}(M) \leqslant 5$, then $6<\widetilde{\mathbf{c}}(M) \leqslant 18$ by (3). For Statement (a), if $2 \leqslant \mu^{-}(M)<4$, (resp. $\mu^{-}(M)=4$ ), the result follows from Theorem 3b (resp. Theorem 3c) of [4]. The 
first two sentences of Statement (b) follows from the classification of all closed connected prime orientable 3-manifolds which admit a contracted triangulation having at most 20 tetrahedra (see [12], §5.4, pp. 224-225). The crystallization of minimal order 18 of $\mathbb{S}^{3} /<2,2,2>$ is depicted in [12], Fig.158, p.198. The crystallization of minimal order 20 of $\mathbb{S}^{3} /<3,2,2>$ is shown in [12], Fig.159, p.198. For lens spaces see [12], §5.4.1, Table 10A. If $5<\mu^{-}(M)<5.2$, then $18<\widetilde{\mathbf{c}}(M)<24$ by (3). Thus the last part of Statement (b) follows from the catalogue of closed orientable 3 -manifolds triangulated by at most 24 colored tetrahedra (see $[5,12])$. The relevant crystallizations of minimal orders 22 and 24 of the spherical manifolds with finite noncyclic fundamental groups are depicted in [12], §5.3.3, pp.199-202. The crystallizations of minimal order 24 of the euclidean fibered manifolds are shown in [12], §5.3.3, pp.202-204. See the Appendix for the list of the manifolds present in the range $5<\mu^{-}(M)<5.2$.

We are going to prove our main result in dimension 4 . We emphasize that the useful topological invariant switches from $\mu^{-}$for $\chi(M)<5$ to $\mu^{+}$for $\chi(M)>5$. It is also remarkable that when $\chi(M)=5$ the distribution of the $\mu(K)$ gives no topological information whatsoever. The trichotomy between $\chi(M)<5, \chi(M)=5$ and $\chi(M)>5$ is striking. It should be interesting to know of other instances where this is important.

Theorem 8. Let $M^{4}$ be a closed connected PL 4-manifold, and $\mathcal{T}_{c}$ the class of all 4dimensional contracted triangulations. Then we have:

(a) If $\chi(M)<5$, then

$$
2 \leqslant \mu^{-}(M)=\frac{5 \widetilde{\mathbf{c}}(M)+10}{7-\chi(M)+\widetilde{\mathbf{c}}(M)}<5=\sup _{K \in \mathcal{T}_{c}} \mu(K)
$$

and equality holds if and only if $M$ is $\mathrm{PL}$ homeomorphic to a 4-sphere. Furthermore, for every $\epsilon>0$ there exists a contracted triangulation $K_{\epsilon}$ of $M$ such that $\mu\left(K_{\epsilon}\right)>5-\epsilon$.

(b) If $\chi(M)=5$, then $\mu^{-}(M)=\mu^{+}(M)=5$.

(c) If $\chi(M)>5$, then

$$
\inf _{K \in \mathcal{T}_{c}} \mu(K)=5<\mu^{+}(M)=\frac{5 \widetilde{\mathbf{c}}(M)+10}{7-\chi(M)+\widetilde{\mathbf{c}}(M)}<6
$$

Furthermore, for every $\epsilon>0$ there exists a contracted triangulation $K_{\epsilon}$ of $M$ such that $\mu\left(K_{\epsilon}\right)<5+\epsilon$.

Proof. Let $(G, c)$ be a crystallization of $M$ of order $p, K=K(G)$ the associated contracted triangulation. Then $\widetilde{\mathbf{c}}(M) \leqslant p-2$. By Lemma (2.1)a [6] we have $q_{3}(K)=(5 p) / 2$ and $q_{2}(K)=\sum_{i<j} g_{i j}$, hence

$$
\mu(K)=\frac{4 q_{3}(K)}{q_{2}(K)}=\frac{10 p}{\sum_{i<j} g_{i j}} .
$$


We now show that $g_{i j} \leqslant p / 2$ for every $i, j \in \Delta_{4}, i \neq j$. Fix $i, j \in \Delta_{4}, i \neq j$, and let $p_{\alpha, i j}$ be the number of vertices of a connected component $C_{\alpha, i j}$ of $G$ colored by $i, j \in \Delta_{4}, i \neq j$, for $\alpha=1, \ldots, g_{i j}$. Then $p_{\alpha, i j}$ is even $(\geqslant 2)$ and $\sum_{\alpha=1}^{g_{i j}} p_{i j}=p$. In fact, the set $\left\{C_{\alpha, i j}\right\}_{\alpha}$ of cardinality $g_{i j}$ induces a partition of the vertex (resp. edge) set of $G$. So we obtain

$$
2 g_{i j} \leqslant \sum_{\alpha=1}^{g_{i j}} p_{i j}=p,
$$

hence $g_{i j} \leqslant p / 2$ for arbitarily fixed distinct colors $i, j \in \Delta_{4}$. This implies that $\sum_{i<j} g_{i j} \leqslant$ $5 p$. Hence the formula in (6) gives $\mu(K) \geqslant 2$ for any contracted triangulation $K$ of $M$. By Lemma (2.2) [6], we have

$$
2 \sum_{r<s<t} g_{r s t}=3 \sum_{i j} g_{i j}-5 p
$$

Substituting (7) into (6) yields

$$
\mu(K)=\frac{30 p}{2 \sum_{r<s<t} g_{r s t}+5 p}<6
$$

as $g_{r s t}>0$ for every distinct colors $r, s, t \in \Delta_{4}$. Thus for every contracted triangulation $K$ of $M$, we have $2 \leqslant \mu(K)<6$, hence $2 \leqslant \mu^{-}(M)<6$.

If $\mu^{-}(M)=2$, then there is a contracted triangulation $K$ of $M$ such that $5 p=\sum_{i<j} g_{i j}$, hence $g_{i j}=p / 2$ for every $i, j \in \Delta_{4}$ and $g_{r s t}=p / 2$ for every $r, s, t \in \Delta_{4}$. Then the 5colored graph $G$ is a dipole of type 5 , that is, it consists of two vertices joined by 5 edges, one of each color in $\Delta_{4}$. This means that the pseudocomplex $K=K(G)$ associated to $G$ consists of two 4-simplexes with identified boundary, so $p=2$ and $\widetilde{\mathbf{c}}(M)=0$ (compare also with [4], p.257). Then $M^{4}$ is PL homeomorphic to a 4-sphere.

By Lemma (2.2) [6] we have

$$
\sum_{i<j} g_{i j}=10+2 p-2 \chi(M)
$$

Substituting (8) into (6) gives

$$
\mu(K)=\frac{5 p}{5-\chi(M)+p} .
$$

Thus for any contracted triangulation $K$ of $M$, we get

$$
2 \leqslant \mu(K)=\frac{5 p}{5-\chi(M)+p}<6 .
$$

Then $\mu(K)$ goes to 5 when $p$ goes to infinity. Note that for any closed connected PL 4 -manifold $M^{4}$ there is a contracted triangulation of arbitrarily large order $p=q_{4}(K)$. It suffices to add a sequence of 2 -dipoles to a crystallization representing $M$. For the notion 
of dipole in a crystallization see the survey papers quoted in Section 1. Given a closed connected PL 4-manifold $M$, the function

$$
f(p)=\frac{5 p}{5-\chi(M)+p}
$$

has derivative with respect to $p$

$$
f^{\prime}(p)=\frac{5(5-\chi(M))}{(5-\chi(M)+p)^{2}}
$$

Then $f(p)$ is an increasing (resp. decreasing) function of $p$ when $\chi(M)<5(\operatorname{resp} . \chi(M)>$ $5)$. For $\chi(M)=5$, then $f(p)=5$ for all $p$, hence $\mu^{-}(M)=\mu^{+}(M)=5$. This proves the statement in (b). For $\chi(M)<5, f(p)$ increases. So the inequality $\widetilde{\mathbf{c}}(M)+2 \leqslant p$, for every $p=q_{4}(K)$, gives

$$
\mu^{-}(M)=\frac{5 \widetilde{\mathbf{c}}(M)+10}{7-\chi(M)+\widetilde{\mathbf{c}}(M)} \leqslant f(p)<5 .
$$

Furthermore, $f(p)$ increasing function and $f(p) \rightarrow 5$ for $p \rightarrow+\infty$ imply the last statement in (a). For $\chi(M)>5, f(p)$ decreases. Then the inequality $\widetilde{\mathbf{c}}(M)+2 \leqslant p$, for every $p$, and $f(p) \rightarrow 5$ for $p \rightarrow+\infty$ imply

$$
\frac{5 \widetilde{\mathbf{c}}(M)+10}{7-\chi(M)+\widetilde{\mathbf{c}}(M)} \geqslant f(p)>5 .
$$

Then $\inf _{K} \mu(K)=5$ and for every $\epsilon>0$ there exists a contracted triangulation $K_{\epsilon}$ of $M$ such that $\mu^{-}(M) \leqslant \mu\left(K_{\epsilon}\right)<5+\epsilon$. Thus $\mu^{+}(M)<6$, and (c) is proved.

Combining Theorem 8 with Theorem 3, we get the following characterizations:

Theorem 9. Let $M^{4}$ be a prime closed connected PL 4-manifold. Then we have:

(a) If $\mu^{-}(M)<\frac{10}{3}$, then $M$ is PL homeomorphic to a 4 -sphere. If $\mu^{-}(M)=\frac{10}{3}$, then $M$ is $\mathrm{PL}$ homeomorphic to either $\mathbb{S}^{1} \times \mathbb{S}^{3}$ or $\mathbb{S}^{1} \times \mathbb{S}^{3}$.

(b) If $\mu^{-}(M)<\frac{80}{21}$ and $\chi(M)=0$, then $M$ is $\mathrm{PL}$ homeomorphic to either $\mathbb{S}^{1} \times \mathbb{S}^{3}$ or $\mathbb{S}^{1} \times \mathbb{S}^{3}$.

(c) If $\mu^{-}(M) \leqslant 4$ and $\chi(M)=1$ or 3 , then $M$ is $\mathrm{PL}$ homeomorphic to either $\mathbb{C} P^{2}$ or $\mathbb{R} P^{4}$.

(d) $\mu^{-}(M) \leqslant \frac{14}{3}$ and $\chi(M)=4$, then $M$ is PL homeomorphic to the topological product $\mathbb{S}^{2} \times \mathbb{S}^{2}$ 
Proof. (a) If $\mu^{-}(M)<\frac{10}{3}$, there is a contracted triangulation $K$ of $M$ such that $\mu(K)<$ $\frac{10}{3}=\frac{2(n+1)}{n-1}$ for $n=4$. Then $M$ is PL homeomorphic to $\mathbb{S}^{4}$ by Theorem 3(b) of [4].

If $\mu^{-}(M)=\frac{10}{3}$, hence $\mu(K)=\frac{10}{3}=\frac{2(n+1)}{n-1}$ for $n=4$, then $M$ is PL homeomorphic to either $\mathbb{S}^{1} \times \mathbb{S}^{3}$ or $\mathbb{S}^{1} \times \mathbb{S}^{3}$ by Theorem $3(\mathrm{c})$ of $[4]$.

(b) If $\chi(M)=0$ and $\tilde{\mu}^{-}(M)<\frac{80}{21}$, from Theorem 8 (a), we get

$$
2 \leqslant \mu^{-}(M)=\frac{5 \widetilde{\mathbf{c}}(M)+10}{7+\widetilde{\mathbf{c}}(M)}<\frac{80}{21}
$$

hence

$$
105 \widetilde{\mathbf{c}}(M)+210<560+80 \widetilde{\mathbf{c}}(M) .
$$

This implies that $\widetilde{\mathbf{c}}(M)<14$. Since $\widetilde{\mathbf{c}}(M)$ is even, it follows that $\widetilde{\mathbf{c}}(M) \leqslant 12$. Now the statement in (b) is proved by using Theorem $3(\mathrm{a}-\mathrm{c})$ and $\chi(M)=0$.

(c) If $\chi(M)=1$ and $\mu^{-}(M) \leqslant 4$, from Theorem 8(a), we obtain

$$
2 \leqslant \mu^{-}(M)=\frac{5 \widetilde{\mathbf{c}}(M)+10}{6+\widetilde{\mathbf{c}}(M)} \leqslant 4
$$

hence $\widetilde{\mathbf{c}}(M) \leqslant 14$. By Theorem $3(\mathrm{~d}) M$ is PL homeomorphic to $\mathbb{R} P^{4}$. If $\chi(M)=3$ and $\mu^{-}(M) \leqslant 4$, from Theorem $8(\mathrm{a})$, we get

$$
2 \leqslant \mu^{-}(M)=\frac{5 \widetilde{\mathbf{c}}(M)+10}{4+\widetilde{\mathbf{c}}(M)} \leqslant 4
$$

hence $\widetilde{\mathbf{c}}(M) \leqslant 6$. By Theorem $3\left(\right.$ a) $M$ is PL homeomorphic to $\mathbb{C} P^{2}$.

(d) If $\chi(M)=4$ and $\mu^{-}(M) \leqslant \frac{14}{3}$, from Theorem 8(a), we obtain

$$
2 \leqslant \mu^{-}(M)=\frac{5 \widetilde{\mathbf{c}}(M)+10}{3+\widetilde{\mathbf{c}}(M)} \leqslant \frac{14}{3}
$$

hence $\widetilde{\mathbf{c}}(M) \leqslant 12$. By Theorem 3(a-c) it follows that $M$ is PL homeomorphic to the topological product $\mathbb{S}^{2} \times \mathbb{S}^{2}$.

Examples. The following are explicit computations of the considered combinatorial invariants for the manifolds listed above:

- Theorem $9(\mathrm{a}, \mathrm{b})$. For $M=\mathbb{S}^{4}$, we have $\mu^{-}(M)=2, \chi(M)=2$ and $\widetilde{\mathbf{c}}(M)=0$. If $M=\mathbb{S}^{1} \times \mathbb{S}^{3}$ or $\mathbb{S}^{1} \times \mathbb{S}^{3}$, then $\mu^{-}(M)=\frac{10}{3}, \chi(M)=0$ and $\widetilde{\mathbf{c}}(M)=8$.

- Theorem 9(c). If $\tilde{M}=\mathbb{C} P^{2}$, then $\mu^{-}(M)=4, \chi(M)=3$ and $\widetilde{\mathbf{c}}(M)=6$. If $M=\mathbb{R} P^{4}$, then $\mu^{-}(M)=4, \chi(M)=1$ and $\widetilde{\mathbf{c}}(M)=14$.

- Theorem $9(\mathrm{~d})$. If $M=\mathbb{S}^{2} \times \mathbb{S}^{2}$, then $\mu^{-}(M)=\frac{14}{3}, \chi(M)=4$ and $\widetilde{\mathbf{c}}(M)=12$.

- The following examples are relevant to the discussion of Theorem 8 as they relate with the $\mu^{+}(M)<6$ portion of the $\chi(M)>5$ inequality. More precisely, we construct two sequences of manifolds that give $\mu^{+} \rightarrow 6$. If $M=h\left(\mathbb{S}^{2} \times \mathbb{S}^{2}\right)$ is the connected sum of $h>1$ copies of $\mathbb{S}^{2} \times \mathbb{S}^{2}$, then $\mu^{+}(M)=\frac{12 h+2}{2 h+1}$ which goes to 6 when $h$ goes to infinity, 
$\chi(M)=2(h+1)$ and $\widetilde{\mathbf{c}}(M)=12 h$. If $M=k\left(\mathbb{C} P^{2}\right), k>1$, then $\mu^{+}(M)=\frac{6 k+2}{k+1}$ which goes to 6 when $k$ goes to infinity, $\chi(M)=k+2$ and $\widetilde{\mathbf{c}}(M)=6 k$. If $M$ is a connected sum of $\ell$ copies of $\mathbb{S}^{1} \times \mathbb{S}^{3}$ and/or $\mathbb{S}^{1} \times \mathbb{S}^{3}$, then $\mu^{-}(M)=\frac{8 \ell+2}{2 \ell+1}$ which goes to 4 when $\ell$ goes to infinity, $\chi(M)=2-2 \ell$ and $\widetilde{\mathbf{c}}(\tilde{M})=8 \ell$.

The following is our main result on the average order for the class of 5-dimensional manifolds (here statement (a) is new, while statements (b) and (c) follow from [4]):

Theorem 10. Let $M$ be a closed connected PL 5-manifold. Then we have

(a)

$$
2 \leqslant \mu^{-}(M) \leqslant \frac{5 \widetilde{\mathbf{c}}(M)+10}{\widetilde{\mathbf{c}}(M)+5 \operatorname{rk}(M)+5}<5
$$

where $\operatorname{rk}(M)$ denotes the rank of the fundamental group $\pi_{1}(M)$. Furthermore, $\mu^{-}(M)=$ 2 if and only if $M$ is PL homeomorphic to $\mathbb{S}^{5}$.

(b) If $\mu^{-}(M)<3$, then $M$ is PL homeomorphic to $\mathbb{S}^{5}$.

(c) If $\mu^{-}(M)=3$, then $M$ is PL homeomorphic to either $\mathbb{S}^{1} \times \mathbb{S}^{4}$ or $\mathbb{S}^{1} \times \mathbb{S}^{4}$.

Proof. Let $(G, c)$ be a crystallization of $M$ with minimum order $p=\widetilde{\mathbf{c}}(M)+2$, and let $K=K(G)$ be the associated contracted triangulation. By Lemma 2.1(a) [8] we get

$$
\mu(K)=\frac{5 q_{4}(K)}{q_{3}(K)}=\frac{15 p}{\sum_{i<j} g_{i j}} .
$$

By Lemma 2.2 and Lemma 2.1(d) of [8], we have

$$
\sum_{i<j} g_{i j}=\sum_{h<k<r<s} g_{h k r s}+3 p-6 \geqslant 3 p+15 \mathrm{rk}(M)+9
$$

On the other hand, $g_{i j} \leqslant p / 2$ for every distinct colors $i, j \in \Delta_{5}$, so we get $\sum_{i<j} g_{i j} \leqslant$ $(15 p) / 2$. Thus we obtain the inequalities in (a). If $\mu^{-}(M)=2$, then there is a contracted triangulation $K$ of $M$ such that $15 p=2 \sum_{i<j} g_{i j}$, hence $g_{i j}=p / 2$ for every $i, j \in \Delta_{5}$. By Lemma 2.2 [8] we get $g_{i j k}=p / 2$ for every distinct colors $i, j, k \in \Delta_{5}$. We see also that $g_{h k r s}=p / 2$ for every 4 -tuple of distinct colors. So the last formula of Lemma 2.2 [8] gives $p=2$. Then $K$ consists of two 5 -simplexes with identified boundary, i.e., $M \cong \mathbb{S}^{5}$. Statements (b) and (c) are those in [4], Theorem 3, for $n=5$.

Examples. The first two inequalities in Theorem 10(a) may become equalities.

For $M=\mathbb{S}^{5}$, we have $\mu^{-}(M)=2$ and $\operatorname{rk}(M)=\widetilde{\mathbf{c}}(M)=0$. If $M=\mathbb{S}^{1} \times \mathbb{S}^{4}$ or $\mathbb{S}^{1} \times \mathbb{S}^{4}$, then $\mu^{-}(M)=3, \operatorname{rk}(M)=1$ and $\widetilde{\mathbf{c}}(M)=10$.

For $M=\mathbb{S}^{3} \times \mathbb{S}^{2}$, we have $\mu^{-}(M)=4.4, \operatorname{rk}(M)=0$ and $\widetilde{\mathbf{c}}(M)=20$. If $M=\mathbb{R} P^{5}$, then $\mu^{-}(M)=4, \operatorname{rk}(M)=1$ and $\widetilde{\mathbf{c}}(M)=30$, as shown in [8], Lemma 1 . 


\section{A question on average order}

The work by Luo and Stong [13] and Tamura [17, 18] implies the following result, stated in $[20]$ :

Theorem 11. For every closed connected topological 3-manifold $M$ and every rational number

$$
4.5<r<6
$$

there is a simplicial triangulation $T$ of $M$ for which the average edge-order $\mu(T)$ is $r$.

The fact that we can find such a triangulation $T$ independent of the topology of $M$ is significant. This is a point of difference with the statement of Theorem 12 (below).

The following question has been posed by Trout in [20]:

Question: Does anyone know of results similar to Theorem 11 but for $n \geqslant 4$ ?

We provide an answer to question above for $n=4$.

Theorem 12. For any rational number

$$
4 \leqslant r<6
$$

there are a closed connected 4-manifold $M$ and a contracted triangulation $K$ of $M$ such that the average 2 -simplex order $\mu(K)$ is $r$.

Proof. First suppose that $4 \leqslant r<5$. Let $r=a / b$, with $a, b>0,(a, b)=1$. Then $a<5 b$ and $4 b \leqslant a$. Let us consider the closed connected 4 -manifold $M=\mathbb{C} P^{2} \# N$, where $N$ is a connected sum of $\ell$ copies of $\mathbb{S}^{1} \times \mathbb{S}^{3}$ and $/$ or $\mathbb{S}^{1} \times \mathbb{S}^{3}$, with $\ell=k(5 b-a)-1$ and $k>1$.

Since $\chi\left(\mathbb{C} P^{2}\right)=3$ and $\chi(N)=2-2 \ell$, it follows that $\chi(M)=3-2 \ell=5-2 k(5 b-a)$. Let $K_{1}$ be a contracted triangulation of $M$ with minimal order $p$, that is, $p=\widetilde{\mathbf{c}}(M)+2$. Then the subadditivity of the reduced complexity implies that

$$
p-2=\widetilde{\mathbf{c}}(M) \leqslant \widetilde{\mathbf{c}}\left(\mathbb{C} P^{2}\right)+\widetilde{\mathbf{c}}(N)=6+8 \ell
$$

hence $p \leqslant 8(\ell+1)=8 k(5 b-a) \leqslant 2 a k$. The last inequality holds as $4 b \leqslant a$ and $k>1$. Adding 2-dipoles (if necessary) to a crystallization representing $K_{1}$, we get a crystallization of order $p+2 n=2 a k$ (recall that $p$ is always even). The last crystallization represents a contracted triangulation $K$ of $M$. Moreover, using (9) we have

$$
\mu(K)=\frac{5(p+2 n)}{5-\chi(M)+p+2 n}=\frac{10 a k}{2 k(5 b-a)+2 a k}=\frac{a}{b}=r .
$$

Suppose now $5 \leqslant r<6$. Then we have $r=a / b, a, b>0,(a, b)=1, a<6 b$ and $5 b \leqslant a$. Let $M$ be the closed connected PL 4-manifold defined as $M=\mathbb{C} P^{2} \# \ell\left(\mathbb{S}^{2} \times \mathbb{S}^{2}\right)$ with $\ell=k(a-5 b)+1$ and $k>1$. Here $\ell\left(\mathbb{S}^{2} \times \mathbb{S}^{2}\right)$ denotes the connected sum of $\ell$ copies of $\mathbb{S}^{2} \times \mathbb{S}^{2}$. Since $\chi\left(\mathbb{C} P^{2}\right)=3$ and $\chi\left(\ell\left(\mathbb{S}^{2} \times \mathbb{S}^{2}\right)\right)=2(\ell+1)$, it follows that $\chi(M)=3+2 \ell=5+2 k(a-5 b)$. Let $K_{1}$ be a contracted triangulation of $M$ with minimal 
order $p$, that is, $p=\widetilde{\mathbf{c}}(M)+2$. Then the subadditivity of the reduced complexity implies that

$$
p-2=\widetilde{\mathbf{c}}(M) \leqslant \widetilde{\mathbf{c}}\left(\mathbb{C} P^{2}\right)+\widetilde{\mathbf{c}}\left(\ell\left(\mathbb{S}^{2} \times \mathbb{S}^{2}\right)\right)=6+12 \ell
$$

hence $p \leqslant 8+12 \ell \leqslant 2 a k$. The last inequality holds as $4+6 \ell \leqslant a k$, that is, $2 \leqslant(6 b-a) k$ which follows from $a<6 b$ and $k>1$. Adding 2-dipoles (if necessary) to a crystallization representing $K_{1}$, we get a crystallization of order $p+2 n=2 a k$. The last crystallization represents a contracted triangulation $K$ of $M$. Moreover, using (9) we have

$$
\mu(K)=\frac{5(p+2 n)}{5-\chi(M)+p+2 n}=\frac{10 a k}{-2 k(a-5 b)+2 a k}=\frac{a}{b}=r .
$$

This completes the proof.

Connections between the average order and the edge diameter of a manifold can be derived from the work by Trout [19].

\section{Appendix}

Here we describe explicitly the closed connected prime orientable PL 3-manifolds $M$ with $5<\mu^{-}(M)<5.2$ completing the statement in the third bullet point of Theorem 7 , part (b). For the theory of Seifert manifolds and for the notation used to describe them we refer to $[14,15]$. However, we recall such a notation to make the reading clear. Let $F$ be a closed connected (orientable $\epsilon=o$ or nonorientable $\epsilon=n$ ) surface of genus $g$. If $b \in \mathbb{Z}$ and $\left(p_{1}, q_{1}\right), \ldots,\left(p_{r}, q_{r}\right)$ are coprime integer pairs with $\left|p_{i}\right| \geqslant 2$, an orientable $(O)$ Seifert manifold $M$ with Seifert invariants

$$
M=\left(O g \epsilon: b\left(p_{1}, q_{1}\right) \cdots\left(p_{r}, q_{r}\right)\right)
$$

is defined as follows. Let $\Sigma$ be $F$ minus $(r+1)$ open discs and let $W$ be the orientable $\mathbb{S}^{1}$-bundle over $\Sigma$. Give $\Sigma$ any orientation, pick a section $\sigma$ of $W$ and choose positive homology bases $\left(\mu_{0}, \lambda_{0}\right),\left(\mu_{1}, \lambda_{1}\right), \ldots,\left(\mu_{r}, \lambda_{r}\right)$ on the components of $\partial W$ arising from the punctures of $F$, with $\mu_{i} \subset \partial \sigma$ and a fibre as $\lambda_{i}$, for every $i=0,1, \ldots, r$. Then $M$ is the Dehn filling of $W$ along $p_{1} \mu_{1}+q_{1} \lambda_{1}, \ldots, p_{r} \mu_{r}+q_{r} \lambda_{r}$, and $\mu_{0}+b \lambda_{0}$. That is, we attach $(r+1)$ solid tori to $W$ along the boundary components sending their meridians to closed simple curves which represent the above-specified homology classes. Then we have

Theorem 13. Let $M$ be a closed connected prime orientable 3-manifold.

- If $5<\mu^{-}(M)<5.2$, then $M$ is homeomorphic to one of the following manifolds:

- Lens spaces: $L(6,1), L(9,2), L(10,3), L(11,3), L(12,5)$, and $L(13,5)$; 
- Spherical manifolds with finite noncyclic fundamental groups (which are Seifert fibered manifolds):

$$
\begin{aligned}
& S^{3} /<3,3,2>\cong(O 0 o:-1(2,1)(3,1)(3,1)) \quad \text { (the octahedral space) } \\
& S^{3} /<5,3,2>\cong(O 0 o:-1(2,1)(3,1)(5,1)) \quad \text { (the Poincaré homology 3-sphere) } \\
& S^{3} /<4,3,2>\cong(O 0 \circ:-1(2,1)(3,1)(4,1)) \\
& S^{3} / \mathbb{Z}_{3} \rtimes_{i} \mathbb{Z}_{8} \cong(O 0 \circ:-1(2,1)(2,-1)(3,1)) \\
& S^{3} / \mathbb{Z}_{5} \rtimes_{i} \mathbb{Z}_{8} \cong(O 0 \text { o: }-1(2,1)(2,1)(5,2)) \\
& S^{3} / \mathbb{Z}_{3} \times Q_{8} \cong(O 0 o:-2(2,1)(2,-1)(2,1)) \\
& S^{3} / Q_{16} \cong(O 0 o:-1(2,1)(2,1)(4,1))
\end{aligned}
$$

- Euclidean fibered manifolds:

$$
\begin{aligned}
& (O 1 n:-1(2,1)(2,1)) \\
& (O 0 o:-1(3,1)(3,1)(3,1)) \\
& (O 0 o:-2(2,1)(2,1)(2,1)(2,1)) \\
& \mathbb{S}^{1} \times \mathbb{S}^{1} \times \mathbb{S}^{1}
\end{aligned}
$$

The relevant crystallizations of minimal order 26 and 28 for closed connected prime $3-$ manifolds can be found in [12], §5.3.5 and $\S 5.3 .6$, pp.205-223. They represent closed 3manifolds $M$ with $5.2 \leqslant \mu^{-}(M) \leqslant 5.25$. For closed 3 -manifolds $M$ with $5.25<\mu^{-}(M) \leqslant$ $\frac{90}{17}$ one can analyze the catalogue produced in [5].

\section{Acknowledgements}

Work performed under the auspicies of the scientific research group G.N.S.A.G.A. of the CNR (National research Council) of Italy and partially supported by the MIUR (Ministero dell'Istruzione, dell'Università e della Ricerca) of Italy within the project Strutture Geometriche, Combinatoria e loro Applicazioni. We are grateful to the anonymous referee for helpful comments and useful suggestions.

\section{References}

[1] P. Bandieri, M.R. Casali and C. Gagliardi. Representing manifolds by crystallization theory: Foundations, Improvements and Related Results. Atti Sem. Mat. Fis. Univ. Modena, Suppl., vol. IL:283-337, 2001.

[2] J. Bracho and L. Montejano. The combinatorics of colored triangulations of manifolds. Geom. Dedicata, 22:303-328, 1987.

[3] M.R. Casali. The average edge order of 3-manifold coloured triangulations. Canad. Math. Bull., 37(2):154-161, 1994. 
[4] M.R. Casali. Average order of coloured triangulations: The general case. Osaka J. Math., 35:249-262, 1998.

[5] M.R. Casali and P. Cristofori. A catalogue of orientable 3-manifolds triangulated by 30 coloured tetrahedra. J. Knot Theory Ram., 17:1-23, 2008.

[6] A. Cavicchioli and F. Spaggiari. Classifying combinatorial 4-manifolds up to complexity. Bol. Soc. Mat. Mexicana, 14(3):303-319, 2008.

[7] A. Cavicchioli and F. Spaggiari. The Combinatorics of Piecewise Linear Manifolds by Colored Graphs. International Mathematical Forum, 7(8):339-368, 2012.

[8] A. Cavicchioli and F. Spaggiari. On reduced complexity of closed piecewise-linear 5-Manifolds. submitted.

[9] A. Cavicchioli, D. Repovš and A.B. Skopenkov. Open problems on graphs arising from geometric topology. Topology Appl., 84:207-226, 1998.

[10] F. Harary. Graph Theory Addison-Wesley, Reading, 1969.

[11] P.J. Hilton and S. Wylie. An Introduction to Algebraic Topology- Homology Theory. Cambridge Univ. Press, Cambridge, 1960.

[12] S. Lins. Gems, Computers and Attractors for 3-Manifolds. Series on Knots and Everything 5 World Scientific Publ., Singapore - New Jersey - London - Hong Kong, 1995.

[13] F. Luo and R. Stong. Combinatorics of triangulations of 3-manifolds. Trans. Amer. Math. Soc., 337(2):891-906, 1993.

[14] J.M. Montesinos. Classical Tessellations and Three-Manifolds. Univesitext, Springer Verlag, Berlin-Heidelberg-New York, 1987.

[15] P. Orlik. Seifert Manifolds. Lecture Notes in Math. 291, Springer Verlag, BerlinHeidelberg-New York, 1972.

[16] C. Rourke and B. Sanderson. Introduction to Piecewise Linear Topology. Springer Verlag, Berlin- Heidelberg- New York, 1969.

[17] M. Tamura. The average edge order of triangulations of 3-manifolds. Osaka J. Math., 33:761-773, 1996.

[18] M. Tamura. The average edge order of triangulations of 3-manifolds with boundary. Trans. Amer. Math. Soc., 350(5):2129-2140, 1998.

[19] A. Trout. Positively curved combinatorial 3-manifolds. The Electronic Journal of Combinatorics, 17:\#R49, 2010.

[20] A. Trout. Mean-number of $n$-simplices per $(n-2)$-simplex in a triangulated $n-$ manifold http://mathoverflow. net/questions/103384 (2013).

[21] A. Vince. n-Graphs. Discrete Math. 72:367-380, 1988.

[22] D. Walkup. The lower bound conjecture for 3- and 4-manifolds. Acta Math., 126:75107, 1970. 